\title{
A new distribution model with two-parameter
}

\author{
Ayse Turan Bugatekin \\ Department of Statistics, Firat University, Elazig, Turkey
}

Received: 23 December 2016, Accepted: 30 January 2017

Published online: 27 April 2017.

\begin{abstract}
This paper deals with a two-parameter new mix distribution called as RL (Rayleigh- Logarithmic) distribution with increasing failure rate. Firstly, probability density function, distribution function, $\mathrm{r}$ th moment, survival and hazard functions of this two-parameter RL distribution are examined. Then, estimation of parameters by using standard statistical techniques such as maximum likelihood method and entropy are given for the new mix model. In the application part, a real data set is receipted and when RL distribution compared with other distributions, this RL distribution is shown be a good competitor.
\end{abstract}

Keywords: Logarithmic distribution, maximum likelihood, Hazard function, Mix distribution, bivariate distributions.

\section{Introduction}

In many cases, known statistical distributions do not sufficient fits to real data. So, several methods for obtained new probability distributions had been studied in the statistical literature recently [13]. Adamidis and Loukas [1] have given the two-parameter exponential -geometric (EG) distribution which has decreasing failure rate. Kus [8] introduced the exponential-Poisson distribution (EP) and examined several of its characteristic properties. Weibull-Poisson (WP) distribution which generalized of the EP distribution was introduced by [11], respectively. Also, Binomial-Exponential 2 distribution is presented by [2].

Increasing failure rate (IFR) distributions are of interest in many real data systems [2]. Cancho et al. [4] obtained Poisson -exponential (PE) distribution with IFR. Also the distributions with IFR had been studied by [9], [3] and [12].

Rayleigh distribution has been commonly used in reliability theory and survival analysis, as its failure rate is a linear function of time. This distribution plays an important role in real life applications since it relates to number distributions such as Weibull and Chi-square distributions. In statistical literature a good amount of work has been devoted to Rayleigh distribution. Several authors such as [6], [14] and the references cited there in have carried out extensive studies as relate to the estimation, prediction and several other inferences with respect to Rayleigh distribution [5].

The purpose of this paper is obtain a new two-parameter mix distribution with IFR properly called as the Rayleigh-Logarithmic (RL) distribution which is constructed as a distribution of independent Rayleigh random variables when the sample size $K$ has a logarithmic distribution. Also, various characteristics of RL distribution and estimation by maximum likelihood and entropy are given.

\section{The new RL distribution.}

Let $Y_{1}, Y_{2}, \ldots, Y_{K}$ be a random sample from a Rayleigh distribution with probability density function (pdf)

$$
f(y, \sigma)=\frac{y}{\sigma^{2}} e^{-y^{2} / 2 \sigma^{2}}, \quad \sigma>0, y \in[0, \infty)
$$




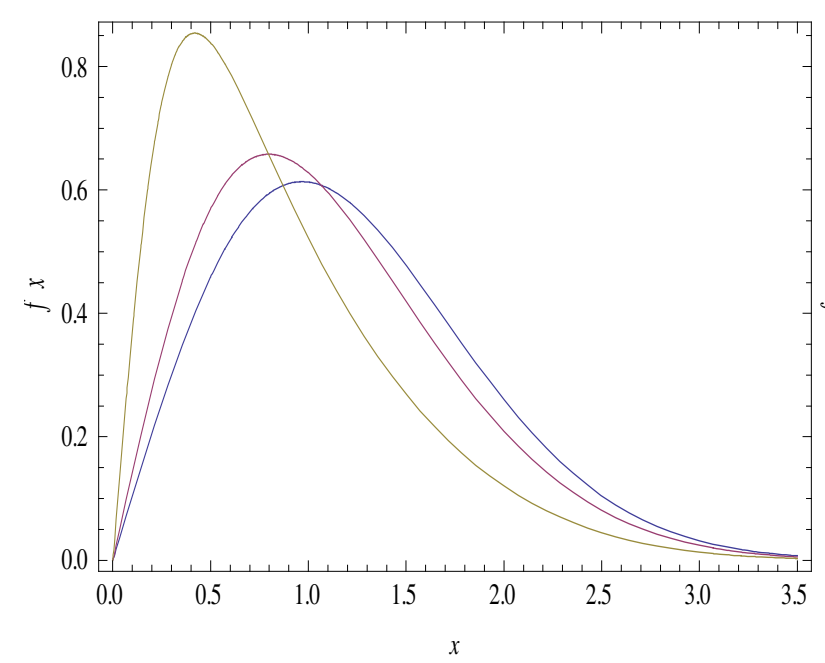

(a)

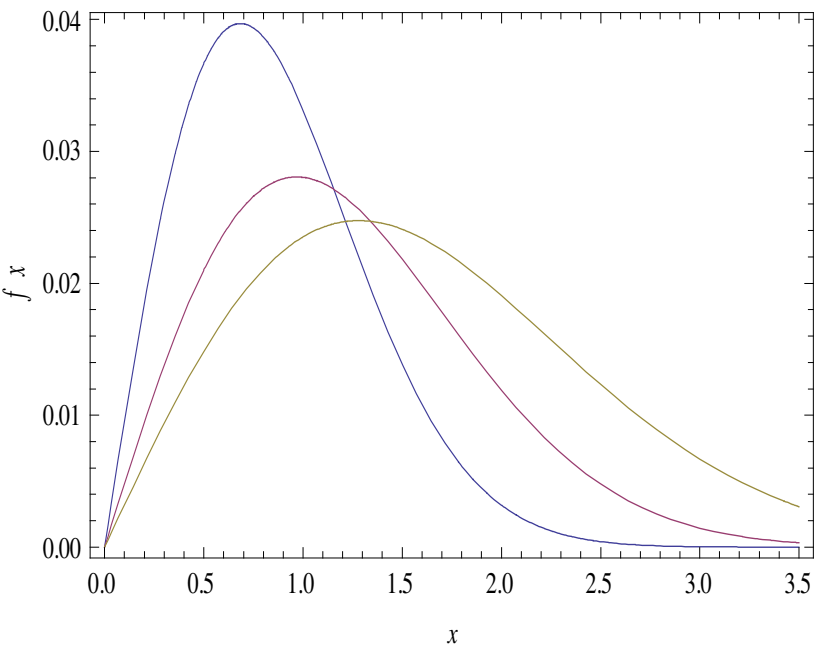

(b)

Fig. 1: (a) Probability Density Functions of RL Distribution for $\sigma^{2}=1, p=0.1,0.5,0.9$, (b) Probability Density Functions of RL Distribution for $p=0.5, \sigma^{2}=0.5,1,1.5$.

and $K$ is a random variable from a logarithmic distribution with probability mass function (pmf)

$$
P(k, p)=-\frac{1}{\ln (1-p)} \frac{p^{k}}{k}, \quad 0<p<1, k \in\{1,2,3, \ldots\}
$$

Let $X=\min \left\{Y_{i}\right\}_{i=1}^{k}$. Then

$$
f(x / k, \sigma)=k \frac{x}{\sigma^{2}} e^{-x^{2} k / 2 \sigma^{2}}
$$

and

$$
f(x, \theta)=-\frac{x}{\sigma^{2} \ln (1-p)} e^{-x^{2} / 2 \sigma^{2}} p\left(1-e^{-x^{2} / 2 \sigma^{2}} p\right)^{-1}, \theta=\left(\sigma^{2}, p\right)
$$

$p d f$ of RL distributions are indicated for different parameter values in Figure 1. Then, this variation is increasing according to increasing value of $\sigma^{2}$.

\section{Statistical and mathematical properties of $\mathrm{RL}$ distribution}

The distribution function of RL distribution is obtained by

$$
F(x, \theta)=1-\frac{\ln \left(1-e^{-x^{2} / 2 \sigma^{2}} p\right)}{\ln (1-p)} .
$$

The moments of $X$ are given by

$$
E\left(X^{r}\right)=-\frac{1}{p \sigma^{2} \ln (1-p)} 2^{r / 2}\left(\sigma^{2}\right)^{-1} \Gamma[1+r / 2] \text { PolyLog }[2+r / 2, p]
$$

where PolyLog(.) is a polylogarithmic function which is really available in standard software such as Mathematica and it is defined as follows [10].

$$
\operatorname{PolyLog}(a, z)=\sum_{k=1}^{\infty} \frac{z^{k}}{k^{a}} .
$$


Therefore the mean and 2 th moment of the RL distribution are obtained, by

$$
E(X)=-\frac{\sqrt{\Pi / 2} \text { PolyLog }[3 / 2, p]}{\ln (1-p)\left(\sigma^{2}\right)^{5 / 2}}
$$

and

$$
E\left(X^{2}\right)=-\frac{2 \text { PolyLog }[2, p]}{\ln (1-p)\left(\sigma^{2}\right)^{3}},
$$

respectively. From here, variance is easily written.

\section{The survival and Hazard functions}

Using $F(x)$, survival function of RL distribution

$$
S(x, \theta)=1-F(x, \theta)=\frac{\ln \left(1-e^{-x^{2} / 2 \sigma^{2}} p\right)}{\ln (1-p)}
$$

and using $F(x)$ and $f(x)$, hazard function of RL distribution

$$
h(x, \theta)=-\frac{x e^{-x^{2} / 2 \sigma^{2}} p\left(1-e^{-x^{2} / 2 \sigma^{2}} p\right)^{-1}}{\sigma^{2} \ln \left(1-e^{-x^{2} / 2 \sigma^{2}} p\right)} .
$$

RL hazard functions for various parameters are indicated in Figure 2. The initial and long-term hazards are $h(0, \theta)=0$ and $h(\infty, \theta)=\infty$, respectively. Thus, the hazard function is a increasing function.

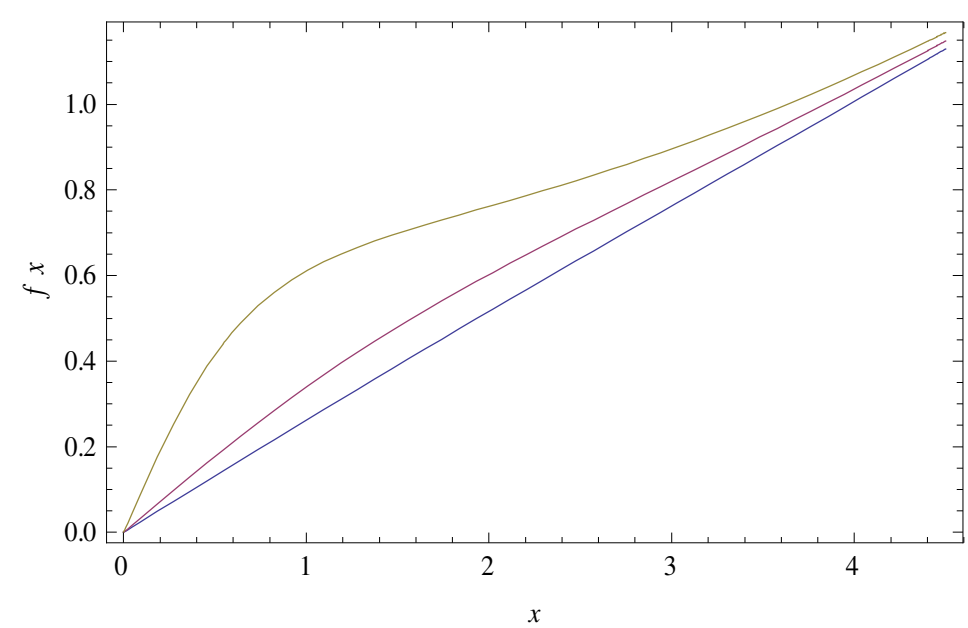

Fig. 2: Hazard Functions of RL Distribution for $\sigma^{2}=4$ and $p=0.1,0.5,0.9$.

\section{Estimation of parameters}

The log-likelihood function of two-parameters RL distribution based on the observed sample size $\mathrm{n}$ is given by

$$
\ell\left(\sigma^{2}, p\right)=\sum_{i=1}^{n} \ln x_{i}+\ln (-1)^{n}-n \ln \sigma^{2}-n \ln (\ln (1-p))-\sum_{i=1}^{n} \frac{x_{i}^{2}}{2 \sigma^{2}}+n \ln p-\sum_{i=1}^{n} \ln \left(1-e^{-x_{i}^{2} / 2 \sigma^{2}} p\right)
$$


and

$$
\begin{gathered}
\frac{\partial \ell}{\partial \sigma^{2}}=\frac{-4 n}{\sigma}+\sum_{i=1}^{n} \frac{x_{i}^{2}}{2 \sigma^{4}}-\sum_{i=1}^{n} \frac{\left(p x_{i}^{2} / 2 \sigma^{4}\right) e^{-x_{i}^{2} / 2 \sigma^{2}}}{\left(1-e^{-x_{i}^{2} / 2 \sigma^{2}} p\right)}=0 \\
\frac{\partial \ell}{\partial p}=\frac{n}{(1-p) \ln (1-p)}+\frac{n}{p}+\sum_{i=1}^{n} \frac{e^{-x_{i}^{2} / 2 \sigma^{2}}}{\left(1-e^{-x_{i}^{2} / 2 \sigma^{2}} p\right)}=0 .
\end{gathered}
$$

\section{Entropy}

The entropy of a random variable $X$ is a measure of uncertainty or randomness of system modeled by this random variable.

If $X$ a random variable with $p d f f(X)$, then its Shannon entropy can be written by $E[-\log f(X)][2]$.

$$
E[-\log f(X)]=\log \frac{p}{\sigma^{2} \ln (1-p)}-E(\log X)+\frac{1}{2 \sigma^{2}} E\left(X^{2}\right)+E\left\{\log \left(1-e^{-x^{2} / 2 \sigma^{2}}\right)\right\}
$$

Then,

and

$$
\begin{gathered}
E(\log X)=\frac{\sigma^{2}\left(\left(\text { EulerGamma }+\log \left[1 /\left(2 \sigma^{2}\right)\right]\right) \log [1-\mathrm{p}]+\operatorname{PolyLog}^{(1,0)}[1, \mathrm{p}]\right)}{2} \\
E\left(X^{2}\right)=-\frac{2 \text { PolyLog }[2, p]}{\ln (1-p)\left(\sigma^{2}\right)^{3}}
\end{gathered}
$$

$$
E\left\{\log \left(1-e^{-x^{2} / 2 \sigma^{2}}\right)\right\}=-\frac{\log [1-\mathrm{p}]^{2}}{2 \mathrm{p} \log [1-\mathrm{p}]}
$$

Hence, Shannon entropy is obtained as

$$
=\log \frac{p}{\sigma^{2} \ln (1-p)}-\frac{\sigma^{2}\left(\left(\text { EulerGamma }+\log \left[1 /\left(2 \sigma^{2}\right)\right]\right) \log [1-\mathrm{p}]+\text { PolyLog }(1,0)[1, \mathrm{p}]\right)}{2}-\frac{\text { PolyLog }[2, p]}{\ln (1-p)\left(\sigma^{2}\right)^{4}}-\frac{\log [1-\mathrm{p}]^{2}}{2 \mathrm{pLog}[1-\mathrm{p}]}
$$

\section{Application}

In practice, a real set which represents the notes of forty-eight students in Mathematics in the final examination of the Indian Institute of Technology, in year 2003 was receipted [7]. Accordingly, the estimated parameters using a maximum likelihood of two-parameter Rayleigh- Logarithmic (RL) distribution were $\hat{p}=0.24$ and $\hat{\sigma}^{2}=372$.

Table 1: Models, estimates of parameters and K-S statistics for data set.

\begin{tabular}{|l|l|l|l|}
\hline Model & $\begin{array}{l}1 \text { th } \\
\text { parameter }\end{array}$ & $\begin{array}{l}2 \text { th } \\
\text { parameter }\end{array}$ & K-S \\
\hline$f_{R L}\left(x ; p, \sigma^{2}\right)=-\frac{x}{\sigma^{2} \ln (1-p)} e^{-x^{2} / 2 \sigma^{2}} p\left(1-e^{-x^{2} / 2 \sigma^{2}} p\right)^{-1}$ & 0.24 & 372 & 0.0864 \\
\hline$f_{B E 2}(x ; \lambda, \beta)=(1+(\beta x-1) \lambda / 2-\lambda) \beta e^{-\beta x}$ & 0.9446 & 0.0732 & 0.0912 \\
\hline$f_{W}(x ; \lambda, \beta)=\lambda \beta^{-\lambda} x^{\lambda-1} e^{-(x / \beta)^{\lambda}}$ & 1.5131 & 28.9154 & 0.1180 \\
\hline$f_{E E}(x ; \lambda, \beta)=\lambda \beta e^{-\beta x}\left(1-e^{-\beta x}\right)^{\lambda-1}$ & 2.5212 & 0.0655 & 0.0937 \\
\hline$f_{W E}(x ; \lambda, \beta)=\beta(\lambda+1) \lambda{ }^{-1} e^{-\beta x}\left(1-e^{-\lambda \beta x}\right)$ & 0.4385 & 3.0740 & 0.0914 \\
\hline$f_{P E}(x ; \lambda, \beta)=\lambda \beta\left(1-e^{-\lambda}\right)^{-1} e^{-\beta x-\lambda e^{-\beta x}}$ & 1 & 0.0743 & $2.3126 \times 10^{-4}$ \\
\hline
\end{tabular}




\section{Conclusions}

In this paper, two-parameter Rayleigh- Logarithmic (RL) mix distribution is introduced. Mathematical and statistical properties of this distribution are given. The estimations of $p$ and $\sigma^{2}$ parameters are examined by maximum likelihood method. Also, entropy of $X$ random variable for measure of uncertainty is calculated and bivariate RL distribution is obtained using marginal distribution functions. In the application part, a real data set is analyzed. As a result, parameters of RL distribution are estimated. Obtained results are compared with other distributions. It is taken Binomial-Exponential 2 (BE2), two-parameter Weibull (W), Exponentiated- Exponential (EE), Weighted- Exponental (WE) and Poisson- Exponential (PE) distributions for compare. It is found that RL distribution is found to be a good competitor according to these 5 distributions for this data set.

\section{Competing interests}

The authors declare that they have no competing interests.

\section{Authors' contributions}

All authors have contributed to all parts of the article. All authors read and approved the final manuscript.

\section{References}

[1] Lewin, L. Polylogarithms and Associated Functions, North Holland Amsterdam, 1981.

[2] Adamidis, K., Loukas, S. A mix Distributions with Decreasing Failure Rate, Statist. Probab. Lett., 39, 35-42, 1998.

[3] Tugrul, O.R. Energy sector and wind energy Potential in Turkey, Renewable Sustainable Energy Rev., 7, 469-484, 2003.

[4] Ross, S.M., Shanthıkumar, J.G., Zhu, Z. On Increasing-Failure-Rate Random Variables, J. Appl. Prob., 42, 797- 809, 2005.

[5] Lariviere, M. A Note on Probability Distributions with Increasing Generalized Failure Rates, Operations Research, 54/3, 602-604, 2006.

[6] Kus, C. A New Lifetime Distribution, Comput. Statist. Data Anal., 51, 4497-4509, 2007.

[7] Eskin, N., Artar, H., Tolun, S. Wind Energy Potential of Gokceada Island in Turkey, Renewable Sustainable Energy Rev. 12, 839-851, 2008.

[8] Brusset, X. Properties of Distributions with Increasing Failure Rate, Munich Personal RePEc Archive, 2009.

[9] Gupta, R.D., Kundu, D. A New Class of Weighted Exponential Distributions, Statistics, 43, 621-634, 2009.

[10] Cancho, V.G., Louzada-Neto, F., Barriga, G.D.C. The Poissson- Exponential Mix Distributions, Comput. Stat. Data Anal., 55, 677-686, 2011.

[11] Lu, W., Shi, D. A New Compounding Life Distribution: The Weibull-Poisson Distribution, J. of Appl. Stat., DOI:10.1080/02664763.2011.575126, 2011.

[12] Silva, R.B., Cordeiro, G.M. The Burr XII Power Series Distributions: A New Compounding Family, Brazilian J. of Prob. and Stat., 29(3), 2013.

[13] Bakouch, H.S., Jazi, M.A., Nadarajah, S., Dolati, A. A Lifetime Model with Increasing Failure Rate, Applied Mathematical Modelling, 38, 5392-5406, 2014.

[14] Dey,S., Dey, T. Statistical Inference for the Rayleigh Distribution under Progressively Type II Censoring with Binomial Removal, Applied Mathematical Modelling, 38, 974-982, 2014. 